\title{
BROADBAND REFLECTARRAYS MADE OF CELLS WITH THREE COPLANAR PARALLEL DIPOLES
}

\author{
Rafael Florencio. Rafael R. Boix, Eduardo Carrasco, \\ José A. Encinar, Mariano Barba, and \\ Gerardo Pérez-Palomino
}

\begin{abstract}
A broadband reflectarray cell made of three parallel dipoles printed on a dielectric layer is presented. A 33\% bandwidth is achieved for the cell made of dipoles, which is larger than that obtained for a reference cell consisting of three stacked square patches $(26 \%)$. Using this cell, a 41 -cm reflectarray antenna has been designed to produce a collimated beam at $9.5 \mathrm{GHz}$. The numerical results obtained for the reflectarray antenna made of parallel dipoles show a $1-d B$ bandwidth of $19 \%$, a $65 \%$ efficiency, $0.2 \mathrm{~dB}$ of losses, and low levels of cross polarization ( $25 \mathrm{~dB}$ below the maximum). These results demonstrate a high performance for the proposed reflectarray antenna made of cells with three printed dipoles.
\end{abstract}

Key words: reflectarrays; broadband antennas; dipole arrays

\section{INTRODUCTION}

Printed reflectarrays are planar reflector antennas made of one or more layers of microstrip patch arrays [1]. Printed reflectarrays present several technological advantages when compared with conventional reflectors and phased arrays such as low cost, low weight, improved polarization performance [2], elimination of complex feed networks [2], possibility of use in dualpolarization applications with different beam shaping in each polarization [3], and so forth.

Some of the first prototypes of reflectarray antennas with elements of variable size were fabricated with one single layer of rectangular patches [2]. Unfortunately, the phase range achieved with the single layer of rectangular elements is usually lower than $330^{\circ}$, and the relation between the phase variation of the reflected field and the size of patches is strongly nonlinear and shows a high slope near resonance [4], which leads to narrow band behavior $[4,5]$. A simple technique to improve the bandwidth is to reduce the period while using a single layer of varying size rectangular patches as proposed in Ref. [6]. However, this technique suffers from some drawbacks: first, the range of phase variation is limited to $300^{\circ}$; and second, it requires very tight photoetching tolerances to realize small gaps 
between adjacent patches. All of these drawbacks have been overcome by the use of multilayered substrates that host either two or three metallization levels of rectangular stacked patches $[5,7]$, or aperture-coupled patches [8], at the cost of a more complex manufacturing process derived from the bonding of different layers with printed elements.

In recent years, different broadband reflectarray elements made of multiresonant elements printed on a single dielectric layer have been proposed to improve the bandwidth, while keeping a simple manufacturing process at the same time. Some of these multiresonant elements on a single layer are parallel dipoles $[9,10]$ and concentric square or cross loops [11-14], where the relative lengths of the dipoles or loops are adjusted to improve the phase range and the bandwidth of the reflectarray element. The use of multiple resonances makes it possible to achieve a variation of phase shift in a range larger than $360^{\circ}$, which can be used as an additional degree of freedom for a further improvement of bandwidth, as demonstrated in Refs. [7]and 8. Different types of printed elements were investigated in Ref. [9], which provided a phase range larger than $600^{\circ}$. However, the novel single-layer reflectarray elements proposed in Ref. [9] lead to phase curves with sharp variations in the slope (which drastically reduces the bandwidth performance), and also, to a rather low antenna efficiency (less than $30 \%$ in the best case for $27 \mathrm{dBi}$ of gain). The element made of three dipoles reported in Ref. [9] was further optimized in Ref. [10] to provide a linear phase response, which made it possible radiation patterns with a $3-\mathrm{dB}$ bandwidth of $13 \%$ around $77 \mathrm{GHz}$ (1-dB bandwidth was $7 \%$ ) for a folded reflectarray antenna based on three closely placed printed dipoles [10]. However, the gain of this antenna was reduced $2 \mathrm{~dB}$ with respect to the conventional rectangular patch antenna because of the higher current density on the narrow strips [10]. Broadband reflectarray elements using double square loops have been proposed in Ref. [11]. Two reflectarray antennas based on these elements demonstrated radiation efficiency close to $52 \%$ and $1-\mathrm{dB}$ gain bandwidth of $9 \%$. However, bandwidth was limited by the nonlinear phase response that occurred for certain dimensions. A practically linear response was achieved by a new reflectarray element that combines rectangle and cross loop elements [12]. A reflectarray using this type of element showed a gain of $33 \mathrm{dBi}$ (that corresponds to $60 \%$ efficiency), a $1-\mathrm{dB}$ gain bandwidth of $24 \%$ and a level of cross polarization $25 \mathrm{~dB}$ below the maximum gain [12], but no information was provided on the losses. An increased 1-dB gain bandwidth of $30 \%$ was obtained for a reflectarray made of concentric cross loops [13] by using an optimization technique for bandwidth enhancement of the type described in Ref. [7]. A single-layer reflectarray cell, which combines a square patch concentric with a square aperture and a varying size concentric loop, called Phoenix cell [14], demonstrated a phase range larger than $600^{\circ}$. However, the phase range is reduced to $360^{\circ}$ to ensure practically parallel phase curves from 15 to $18 \mathrm{GHz}$ ( $18 \%$ bandwidth) and very low losses (around $0.05 \mathrm{~dB}$ ). In addition, no information is provided in Ref. [14] on the cross polarization produced by the Phoenix element on the antenna performance.

In all previous references dealing with novel single-layer reflectarray elements, the work is focused on bandwidth improvement, but there is not a complete information on other antenna characteristics, such as losses and cross polarization. In a real application, the reflectarray performance has to be compliant with the requirements of low losses, low cross polarization, high efficiency, and bandwidth. Several reflectarrays made of three layers of varying-size patches have provided very good

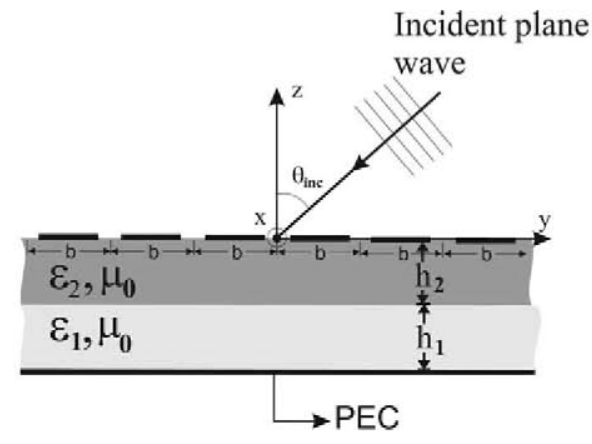

(a)

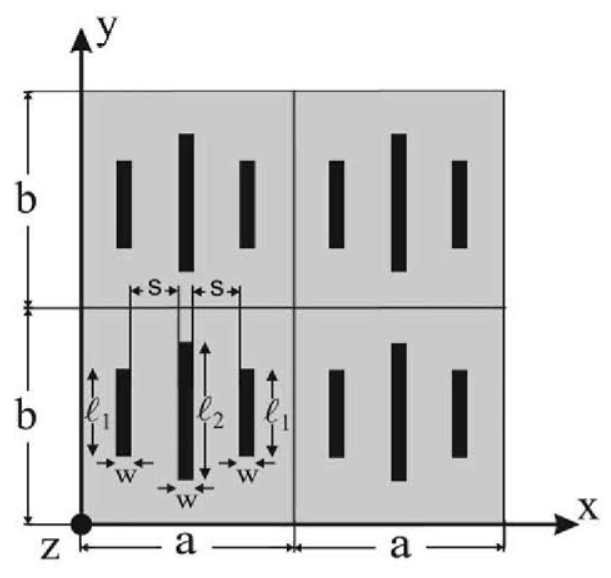

(b)

Figure 1 Two-layered periodic structure with three parallel dipoles in the unit cell. The periodic structure is illuminated by a plane wave. (a) Side view and (b) top view

performance in terms of bandwidth, losses, and cross polarization for real applications $[3,15]$. The goal of the this article is to demonstrate that a simple reflectarray element made of three parallel dipoles can provide a performance similar to that of the well-known element made of three stacked patches in terms of bandwidth, losses, and cross polarization. It should be pointed out that the reflectarrays made of parallel dipoles have the additional advantage that their manufacturing process is simpler than that of reflectarrays made of stacked patches, which obviously implies a reduction in the production costs.

\section{EXPERIMENTAL VALIDATION OF THE NUMERICAL CODE FOR THE ANALYSIS OF PERIODIC ARRAYS OF THREE PARALLEL DIPOLES}

The unit cell considered in this work is made of three parallel dipoles printed on a dielectric layer above a ground plane. When choosing the sizes of the dipoles of the reflectarray that lead to the appropriate reflection phases, we will assume that each element comprising three dipoles is immersed in an infinite periodic array of elements of the same size [2]. This is known as local periodicity assumption [1], and it makes it possible to design reflectarray antennas within reasonable CPU times $[2,3$, 5]. The validity of the local periodicity assumption is based on the fact that it leads to numerical results that show good agreement with experimental results $[2,3,5,15]$. Figure 1 shows the side and top views of four identical reflectarray cells. The parallel dipoles are printed on a substrate comprising two layers of 


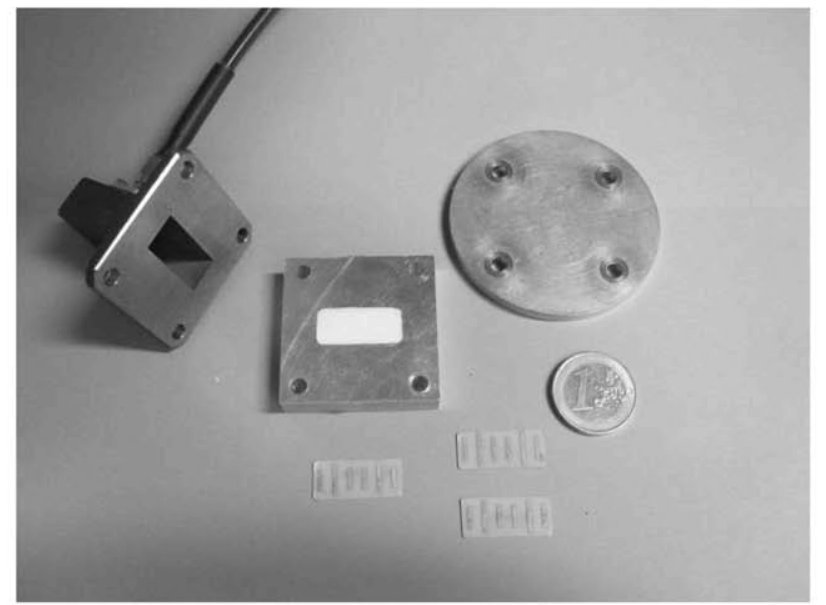

Figure 2 Samples used for measurements in WR-90 WGS

thickness $h_{i} \quad(i=1,2) \quad$ and complex permittivity $\varepsilon_{i}=\varepsilon_{0} \varepsilon_{r i}\left(1-j \tan \delta_{i}\right) \quad(i=1,2)$. The periodic structure is illuminated by a plane wave, and the incidence direction of the plane wave is given by the angular spherical coordinates $\theta_{\text {inc }}$ and $\phi_{\text {inc }}$. Assuming the formation of grating lobes is avoided in the periodic structure, let $\mathbf{E}^{\text {inc }}(x, y, z>0)$ be the electric field of the plane wave impinging on the periodic structure in the upper half space of Figure 1, and let $\mathbf{E}^{\mathrm{ref}}(x, y, z>0)$ be the electric field of the reflected wave. Let us define a scattering matrix $\overline{\mathbf{S}}_{X Y}$ that relates the complex amplitudes of the tangential components of

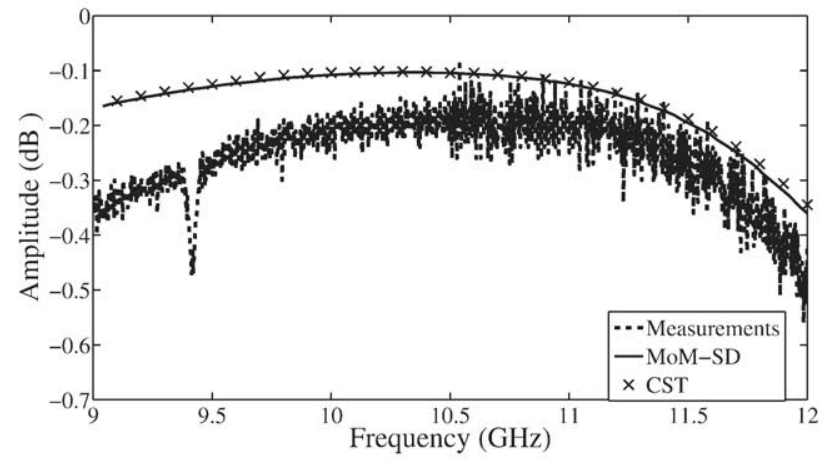

(a)

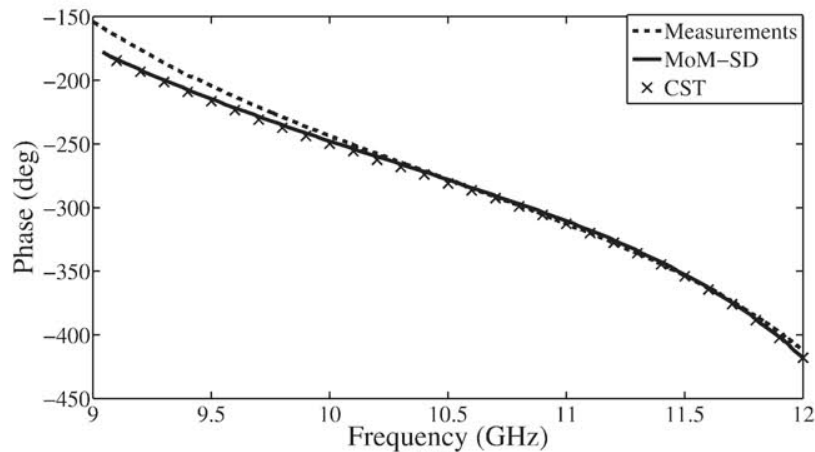

(b)

Figure 3 Numerical results and WGS measurements obtained for the magnitude (a) and phase (b) of $S_{y y}$. Parameters: $a=11.43 \mathrm{~mm}$; $b=10.16 \mathrm{~mm} ; l_{2}=9 \mathrm{~mm} ; l_{1}=0.7 l_{2} ; s=2.81 \mathrm{~mm} ; w=1 \mathrm{~mm} ;$ $\varepsilon_{r 1}=1.067 ; \tan \delta_{1}=0.0002 ; \quad h_{1}=3 \mathrm{~mm} ; \varepsilon_{r 2}=3.38 ; \tan \delta_{2}=0.005 ;$ $h_{2}=0.508 \mathrm{~mm}$
$\mathbf{E}^{\text {ref }}\left(x, y, z=0^{+}\right)$and $\mathbf{E}^{\text {inc }}\left(x, y, z=0^{+}\right)$(i.e., the complex amplitudes of the tangential reflected and incident electric fields at the upper limit of the multilayered substrate) as shown below

$$
\left(\begin{array}{c}
E_{0 x}^{\mathrm{ref}} \\
E_{0 y}^{\mathrm{ref}}
\end{array}\right)=\overline{\mathbf{S}}_{X Y} \cdot\left(\begin{array}{l}
E_{0 x}^{\mathrm{inc}} \\
E_{0 y}^{\mathrm{inc}}
\end{array}\right)=\left(\begin{array}{ll}
S_{x x} & S_{x y} \\
S_{y x} & S_{y y}
\end{array}\right) \cdot\left(\begin{array}{c}
E_{0 x}^{\mathrm{inc}} \\
E_{0 y}^{\mathrm{inc}}
\end{array}\right) .
$$

The phase of the diagonal coefficient $S_{y y}$ is the key parameter in the design of a reflectarray antenna. A numerical code has been implemented which makes it possible to compute the scattering matrix $\overline{\mathbf{S}}_{X Y}$. This numerical code makes use of the method of moments in the spectral domain (MoM-SD) based on multilayered Green's function (MGF) [16]. In this section, the results obtained with the numerical code are validated by comparing with measurements carried out in a waveguide simulator (WGS) [17], and with results provided by the commercial software CST $^{\circledR}$ [18]. The photograph of Figure 2 shows the experimental setup used in the WGS measurements. Two periodic cells of parallel dipoles (see Fig. 1) are printed on $0.508 \mathrm{~mm}$ thick an Arlon ${ }^{\circledR}$ layer $\left(\varepsilon_{r 2}=3.38 ; \tan \delta_{2}=0.005\right)$, placed on top of a 3 $\mathrm{mm}$ thick, Rohacell ${ }^{\circledR}$ layer $\left(\varepsilon_{r 1}=1.067 ; \tan \delta_{1}=0.0002\right)$, and the whole two-layered structure with two periodic cells is enclosed within a WR-90 waveguide for measurements in the $\mathrm{X}$-band. The reflection coefficient of the short-circuited waveguide section shown in Figure 2 is measured using a network analyzer. Then, taking into account the relationship between frequency and angle of incidence in the WGS, the corresponding reflection coefficients are generated with our numerical code and with $\mathrm{CST}^{\circledR}$.

Figure 3 shows the simulated and measured results for the magnitude and phase of $S_{y y}$. Note there is an excellent agreement between our numerical results and the numerical results provided by $\operatorname{CST}^{\circledR}$. It should be pointed out that our home-made software is roughly 500 times faster than $\operatorname{CST}^{\circledR}$ [16], which makes it possible to design reflectarray antennas under the local periodicity condition with our software within reasonable CPU times (the design of a reflectarray antenna involving optimization at different frequencies may require the analysis of hundreds of thousands of periodic structures [3]). The agreement between the measured and simulated phase shift is excellent, except at very low frequencies where the effect of the equivalent dipoles in a real reflectarray would be practically negligible. There are also some discrepancies between measured and simulated losses [see Fig. 3(a)]. These discrepancies are attributed to small tolerances in the complex dielectric constant of the materials, and also to the moisture present in the Rohacell ${ }^{\circledR}$ samples, which may have increased the losses above the expected level.

\section{BANDWIDTH FOR THE ELEMENT WITH THREE PARALLEL DIPOLES}

In this section, we carry out a comparison between the reflectarray element containing three parallel dipoles and the reflectarray element containing three stacked square patches. The comparison focuses on the bandwidth performance of both elements. The authors of Ref. [4] define the bandwidth of a reflectarray element as the frequency interval where the phase of the reflection coefficient does not differ from the phase at the center frequency $f_{0}$ more than $45^{\circ}$ for any value of the geometrical parameter used for the phase variation. In this article, we are going to use an alternative definition which is based on the ideal reflectarray element concept. According to Ref. [1] (p. 95), the ideal reflectarray element should produce a phase shift in the reflected electric field which varies linearly with frequency $f$ 


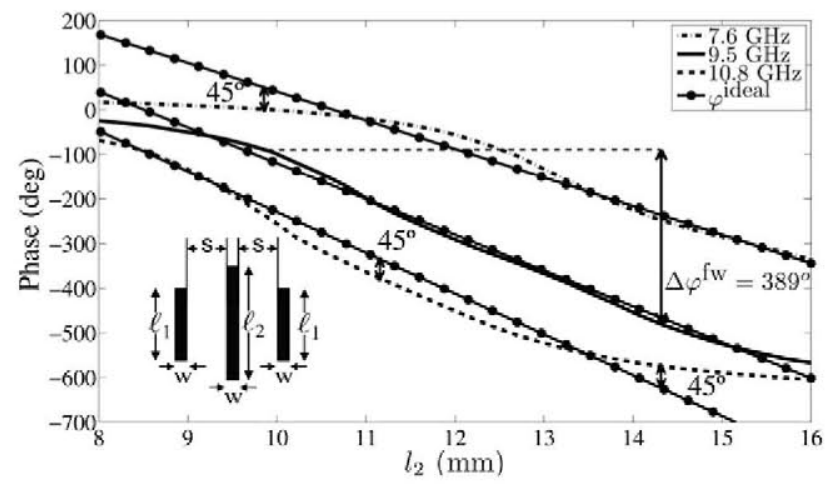

(a)

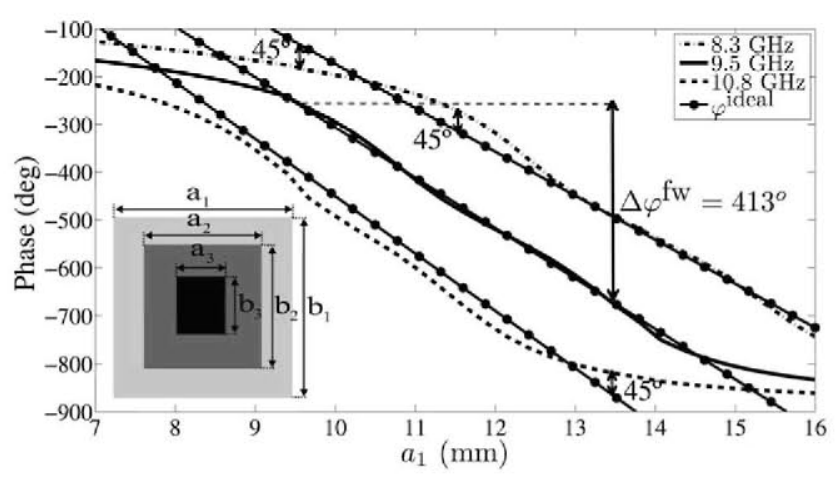

(b)

Figure 4 Phase and magnitude of $S_{y y}$ for different reflectarray elements. (a) Phase for the element consisting of three parallel dipoles on a two-layered substrate (see Fig. 1). (b) Phase for the element consisting of three stacked square patches where every patch is printed on the same two-layered substrate used for the dipoles

and with the geometrical parameter $l$ used for the phase adjustment, that is, the phase of the ideal reflectarray element $\varphi^{\text {ideal }}$ should be a function of $f$ and $l$ given by:

$$
\varphi^{\text {ideal }}(f, l)=\alpha+\beta f l
$$

where $\alpha$ and $\beta$ are constant coefficients. Two different reflectarray elements (one containing three parallel dipoles and the other containing three stacked square patches) have been designed to provide a linear phase response in a wide frequency band by adjusting different geometrical parameters. The results are compared in Figures 4(a) and 4(b) for normal incidence. In all cases the period is $a=b=16.5 \mathrm{~mm}$. For the reflectarray element with three parallel dipoles, the metallizations are printed on an Arlon $^{(3)}$ layer $0.508 \mathrm{~mm}$ thick placed on top of a Rohacell ${ }^{(3)}$ layer $3 \mathrm{~mm}$ thick, and the dimensions of the dipoles are $l_{1}=0.7 l_{2}$, $s=4.5 \mathrm{~mm}$, and $w=1 \mathrm{~mm}$ [see Fig. 4(a)]. For the reflectarray element based on three stacked square patches, every patch is printed on an Arlon ${ }^{(3)}$ layer $0.508 \mathrm{~mm}$ thick placed on top of a Rohacell ${ }^{\circledR 2}$ layer $3 \mathrm{~mm}$ thick. In this latter case, the patch dimensions are $a_{1}=b_{1}, a_{2}=b_{2}=0.9 \quad a_{1}$, and $a_{3}=b_{3}=0.7 \quad a_{1}$ [see Fig. 4(b)]. Figures 4(a) and 4(b) show the phase curves of $S_{y y}$ for the two reflectarray elements as a function of $l\left[l=l_{2}\right.$ in Fig. 4(a) and $l=a_{1}$ in Fig. 4(b)] for different frequencies. All these phase curves have been obtained by means of the MoM-SD based on MGFs [16]. To determine the phase of the ideal reflec- tarray element at each frequency, we have first carried out a least squares linear fitting of the phase curve at the center frequency $f_{0}\left[f_{0}=9.5 \mathrm{GHz}\right.$ in Figs. 4(a) and 4(b)], and we have subsequently extracted the coefficients $\alpha$ and $\beta$ from this linear fitting by using Eq. (2). Once $\alpha$ and $\beta$ are available, the phase of the ideal reflectarray element at frequencies different from $f_{0}$ has been obtained by means of (2) (a similar strategy has been followed in Ref. [8]). When the phase of the ideal reflectarray element is known at all frequencies, in this work the bandwidth of the reflectarray element is defined as the frequency interval $f_{1}<f<f_{2}$ where the simulated phase of the element does not differ from the phase of the ideal element more than $45^{\circ}$ in a range of values of $l$ covering a phase range $\Delta \varphi^{\mathrm{fw}}$ of at least $360^{\circ}$ at the center frequency $f_{0}$ (this range of values of $l$ is chosen within the range for which the phase curve at $f_{0}$ more closely matches the straight line representing the phase of the ideal element). Although a value of $\Delta \varphi^{\mathrm{fw}}$ of at least $360^{\circ}$ cannot be reached with all types of reflectarray elements [4], it can be achieved with the broadband reflectarray elements that have been particularly studied in Figures 4(a) and 4(b). With the new definition given in this article for the bandwidth of a reflectarray element, the element based on three parallel dipoles of Figure 4(a) shows a bandwidth of $33.7 \%\left(f_{2}-f_{1}=3.2 \mathrm{GHz}\right)$ along the range $9.9 \mathrm{~mm}<l=l_{2}<14.3 \mathrm{~mm}$ while covering a phase range $\Delta \varphi^{\mathrm{fw}}=389^{\circ}$ along that range of $l$ at $f_{0}=9.5 \mathrm{GHz}$. The reflectarray element based on three stacked square patches of Figure $4(\mathrm{~b})$ shows a bandwidth of $26.3 \%\left(f_{2}-f_{1}=2.5 \mathrm{GHz}\right)$ along the range $9.7 \mathrm{~mm}<l=a_{1}<13.4 \mathrm{~mm}$ while covering a phase range $\Delta \varphi^{\mathrm{fw}}=413^{\circ}$ along that range of $l$ at $f_{0}=9.5 \mathrm{GHz}$. These results demonstrate that the reflectarray element based on three parallel dipoles shows a bandwidth and a phase range that are similar to those provided by the reflectarray element based on three stacked square patches. However, the element based on paralell dipoles has the additional advantage that its manufacturing process is simpler and cheaper than that of the element based on three stacked patches (whereas the reflectarray based on parallel dipoles only involves one single level of metallization, the reflectarray based on stacked patches involves three superimposed levels of metallization).

\section{DESIGN OF REFLECTARRAY ANTENNAS BASED ON THE ELEMENT WITH THREE PARALLEL DIPOLES}

In Section 3 we demonstrated that the bandwidth performance of the reflectarray element based on three parallel dipoles is slightly better than to that of the element based on three stacked square patches. To check if the bandwidth performance of the elements also applies to the bandwidth performance of the reflectarrays, we have designed two collimated beam reflectarrays using the reflectarray elements defined in the previous section, one made of parallel dipoles and one made of stacked square patches. The reflectarrays have been designed to radiate a main beam in the direction $\theta_{0}=18.9^{\circ}$ and $\phi_{0}=90^{\circ}$ at 9.5 $\mathrm{GHz}$, where $\theta_{0}$ and $\phi_{0}$ are the conventional spherical coordinates defined in Ref. [5]. The reflectarrays are circular and consist of 489 elements arranged in a $25 \times 25$ grid with cell size $16.5 \times 16.5 \mathrm{~mm}^{2}$. In both reflectarrays, the phase center of the feed is assumed to be located at the point of coordinates $x=0 \mathrm{~mm}, y=-116 \mathrm{~mm}$, and $z=340 \mathrm{~mm}$ with respect to the center of the reflectarrays. The radiation pattern of the feed is modeled as a function $\cos ^{9}(\theta)$, which provides an illumination level at the reflectarray edges $12 \mathrm{~dB}$ below the maximum. In the design of the two collimated beam reflectarrays, the geometrical dimensions of the elements that produce the required phase shift in each reflectarray cell are adjusted by iteratively calling 


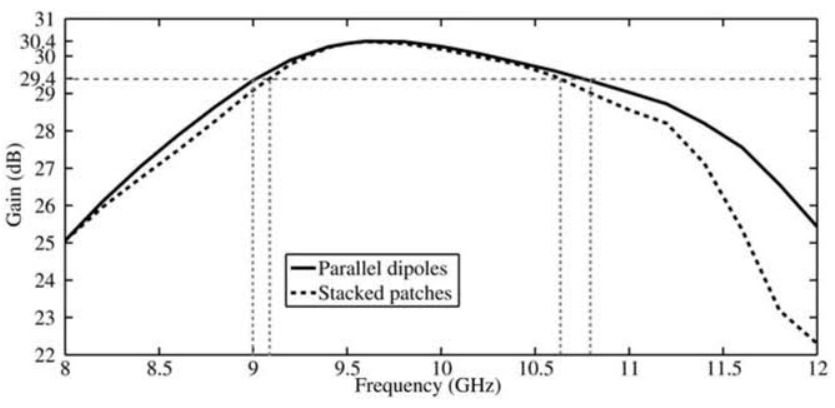

Figure 5 Variation of the gain with frequency for both the reflectarray antenna made of dipoles and the reflectarray antenna made of stacked patches

the analysis routine while considering the real angles of incidence [5]. Once all the element dimensions have been determined, each reflectarray cell is analyzed by the MoM-SD software. Then, the radiation patterns and the antenna gain are obtained from the matrix $\overline{\mathbf{S}}_{X Y}$ computed for each element as described in Ref. [1] (pp. 68-72). Figure 5 shows the results obtained for the variation of the gain of the two reflectarray antennas as a function of frequency. Note that the antenna made of parallel dipoles presents a bandwidth of $19.0 \%$ when the gain variation is limited to $1 \mathrm{~dB}$ below the maximum gain. The antenna made of stacked patches shows a bandwidth of $16.4 \%$ for a gain variation of $1 \mathrm{~dB}$, which is slightly smaller than the bandwidth of the antenna made of parallel dipoles. Note that the antenna bandwidth is smaller than the bandwidth obtained for the reflectarray element in both configurations. The reasons are: first, because the definition of 1-dB bandwidth for the antenna is more stringent than the $45^{\circ}$ definition used in the phase of the reflectarray element; and second, because whereas the element bandwidth is only evaluated at normal incidence, the

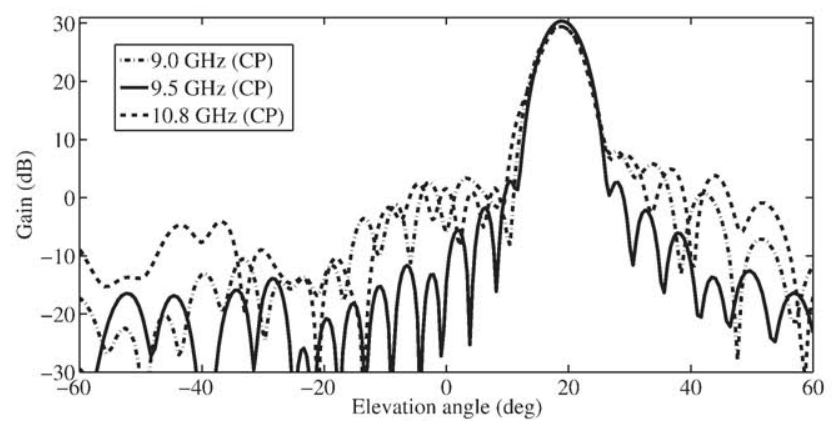

(a)

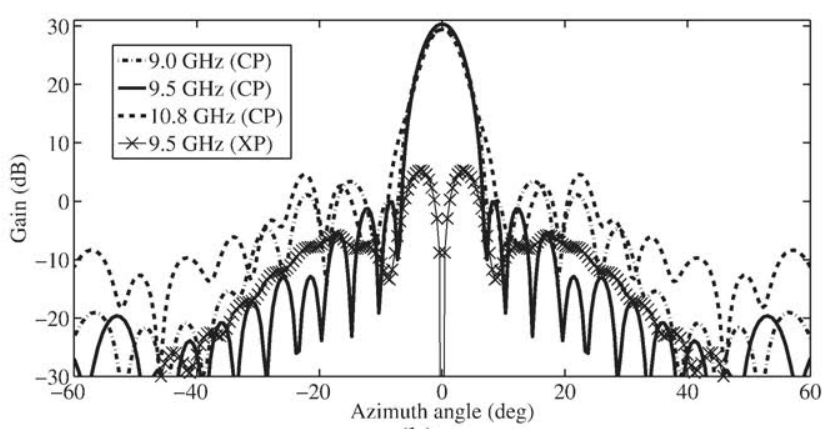

(b)

Figure 6 Radiation patterns in elevation plane (a) and azimuth plane (b) for a collimated beam reflectarray antenna based on elements with three parallel dipoles. CP and XP components are shown

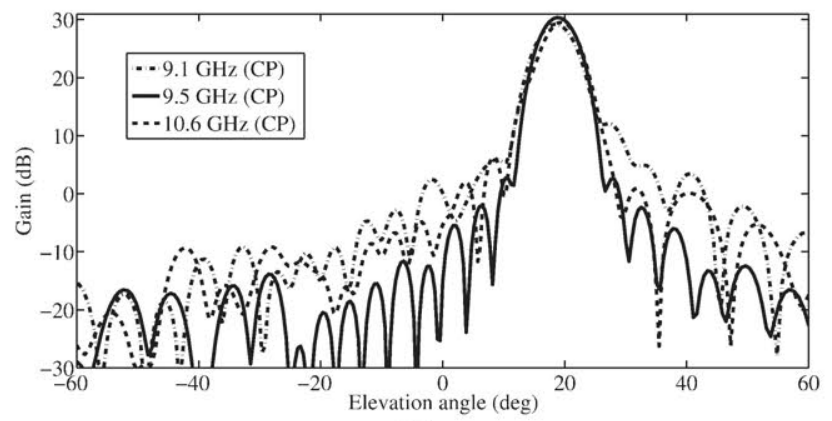

(a)

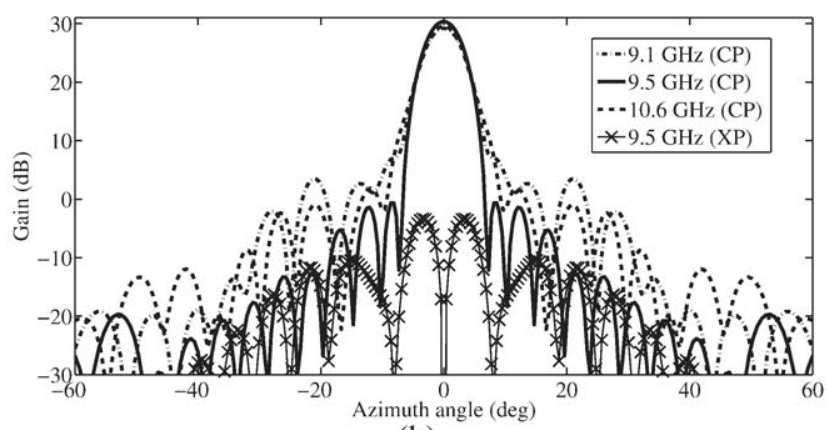

(b)

Figure 7 Radiation patterns in elevation plane (a) and azimuth plane (b) for a collimated beam reflectarray antenna based on elements with three stacked square patches. CP and XP components are shown

antenna bandwidth is obtained by considering the real angle of incidence on each reflectarray element. Note that the bandwidth is larger for the antenna made of dipoles in accordance with the results obtained for the element made of dipoles in Section 3. The 1-dB bandwidth obtained for the reflectarray made of parallel dipoles is $19.0 \%$, which is similar to the bandwidths obtained for other single-layer reflectarray elements proposed for broadband operation without optimization [12, 14]. Note that in this work the antenna was designed only at the center frequency as in Refs. [12] and [14], and therefore, a further improvement can be achieved by optimizing the dipole lengths with an optimization tool that enforces the phase requirements at different frequencies as in Refs. [3, 7, 13], and [15]. The simulated antenna gain at the center frequency $(9.5 \mathrm{GHz})$ is $30.4 \mathrm{~dB}$, which represents a $65 \%$ efficiency. Note that this efficiency is comparable to those obtained in reflector antennas, and it is higher than the efficiency obtained by other single-layer reflectarrays [10-12]. The losses on the reflectarray antenna are computed as the difference between the simulated gain considering dielectric losses and the gain resulting from the ideal phase distribution. The antenna losses turn out to be $0.2 \mathrm{~dB}$.

Figures 6 and 7 show the copolar (CP) radiation patterns in the principal planes for the two designed collimated beam reflectarrays at the center frequency $9.5 \mathrm{GHz}$, and at the limits of the frequency interval for which the antenna gain is $1 \mathrm{~dB}$ below the maximum gain. It can be seen that the main beam of the radiation patterns is reasonably well preserved. In the case of the reflectarray made of dipoles, the side lobes are kept at 20 $\mathrm{dB}$ below the maximum in the elevation plane, and $25 \mathrm{~dB}$ below the maximum in the azimuth plane. Figures 6(b) and 7(b) show that the maximum cross-polar (XP) radiation in the azimuth plane at $9.5 \mathrm{GHz}$ is $25 \mathrm{~dB}$ below the main beam $\mathrm{CP}$ radiation in the case of the reflectarray made of dipoles, and $34 \mathrm{~dB}$ below the main beam $\mathrm{CP}$ radiation in the case of the reflectarray made 
of stacked patches [similar cross-polarizations levels have been found in the azimuth plane at the limits of the frequency band for a gain variation of $1 \mathrm{~dB}$, but they are not shown in Figs. $6(\mathrm{~b})$ and $7(\mathrm{~b})]$. The cross-polarization radiation in the elevation plane [see Figs. 6(a) and 7(a)] is lower than $-30 \mathrm{dBi}$ because the elevation plane is a symmetry plane. These results show low levels of cross polarization for both reflectarrays.

\section{CONCLUSION}

WGS measurements and the commercial software $\operatorname{CST}^{\circledR}$ have been used to validate a MoM-SD home-made software for the analysis of a multilayered periodic structure containing three parallel dipoles in the unit cell. The MoM-SD software has been used to prove that the bandwidth of the reflectarray element based on three parallel dipoles is larger than that of the reflectarray element based on three stacked square patches. Also, the bandwidth and cross-polarization performance of a reflectarray antenna made of parallel dipoles have been compared to that of a reflectarray antenna made of stacked square patches. The results obtained show that the antenna made of dipoles provides a high performance in terms of bandwidth, losses, and efficiency, while the manufacturing costs are smaller than those of the antenna made of stacked patches. Although the reflectarray based on dipoles was designed for one single polarization, this design can be extended to dual-polarization applications by printing a second array of orthogonal dipoles at the opposite side of the layer containing the original dipoles. These orthogonal dipoles would make it possible to adjust the phasing for the orthogonal polarization. The antenna bandwidth for either single or dual polarization can be further improved if the dipole dimensions are adjusted by means of an optimization tool that enforces the phase requirements at different frequencies.

\section{ACKNOWLEDGMENT}

This work has been supported by "Junta de Andalucía" (project P09-TIC-4595) and by the Spanish Ministry of Science and Innovation (projects CICYT TEC2010-17567 and CONSOLIDER CSD2008-68).

\section{REFERENCES}

1. J. Huang and J.A. Encinar, Reflectarray antennas, IEEE Press/Wiley, Piscataway, New Jersey/New York, 2008.1.

2. D.M. Pozar, S.D. Targonski, and H.D. Syrigos, Design of millimeter wave microstrip reflectarray, IEEE Trans Antennas Propag 45 (1997), 287-296.

3. J.A. Encinar, L. Datashvili, J.A. Zornoza, M. Arrebola, M. SierraCastañer, J.L. Besada, H. Baier, and H. Legay, Dual-polarization dual-coverage reflectarray for space applications, IEEE Trans Antennas Propag 54 (2006), 2828-2837.

4. M. Bozzi, S. Germani, and S. Perregrini, Performance comparison of different element shapes used in printed reflectarrays, IEEE Antennas Wireless Propag Lett 2 (2003), 219-222.

5. J.A. Encinar, Design of two-layer printed reflectarrays using patches of variable size, IEEE Trans Antennas Propag 49 (2001), 14031410.

6. D.M. Pozar, Wideband reflectarrays using artificial impedance surfaces, Electron Lett 43 (2007), 148-149.

7. J.A. Encinar and J.A. Zornoza, Broadband design of three-layer printed reflectarrays, IEEE Trans Antennas Propag 51 (2003), 16621664.

8. E. Carrasco, J.A. Encinar, and M. Barba, Bandwidth improvement in large reflectarrays by using true-time-delay, IEEE Trans Antennas Propag 56 (2008), 2496-2503.

9. S. Dieter, C. Fischer, and W. Menzel, Single-layer unit cells with optimized phase angle behaviour, In: Proceedings of the 3rd Euro- pean Conference on Antennas and Propagation, EuCAP 2009, March 23-27, 2009, pp. 1149-1153.

10. W. Menzel, L. Jiang, and S. Dieter, Folded reflectarray antenna based on a single layer reflector with increased phase angle range, In: Proceedings of the 3rd European Conference on Antennas and Propagation, EuCAP 2009, March 23-27, 2009, pp. 2757-2760.

11. M.R. Chaharmir, J. Shaker, M. Cuhaci, and A. Ittipiboon, A broadband reflectarray antenna with double square rings, Microwave $\mathrm{Opt}$ Technol Lett 48 (2006), 1317-1320.

12. M.R. Chaharmir and J. Shaker, Broadband reflectarray with combination of cross and rectangle loop elements, Electron Lett 44 (2008), $658-659$

13. M.R. Chaharmir, J. Shaker, and H. Legay, Broadband design of a single layer reflectarray using multi cross loop elements, IEEE Trans Antennas Propag 57 (2009), 3363-3366.

14. L. Moustafa, R. Gillard, F. Peris, R. Loison, H. Legay, and E. Girard, The Phoenix cell: A new reflectarray cell with large bandwidth and rebirth capabilities, IEEE Antennas Wireless Propag Lett 10 (2011), 71-74

15. J.A. Encinar, M. Arrebola, L.F. de la Fuente, and G. Tosso, A transmit receive reflectarray antenna for direct broadcast satellite applications, IEEE Trans Antennas Propag 59 (2011), 3255-3264.

16. R. Florencio, R.R. Boix, E. Carrasco, J.A. Encinar, and V. Losada, Efficient numerical tool for the analysis and design of reflectarrays based on cells with three parallel dipoles, Microwave Opt Technol Lett 55 (2013), 1212-1216.

17. P.W. Hannan and M.A. Balfour, Simulation of a phased-array antenna in waveguide, IEEE Trans Antennas Propag 13 (1965), 342353.

18. CST Microwave Studioß), Computer Simulation Technology, Wellesley Hills, MA 02481. Available at: www.cst.com. 